University of Nebraska - Lincoln

DigitalCommons@University of Nebraska - Lincoln

$11-1-1999$

\title{
Confined water in hydrophobic nanopores: Dynamics of freezing into bilayer ice
}

\author{
Jan Slovak \\ Okayama University \\ Kenichiro Koga \\ Okayama University, koga@cc.okayama-u.ac.jp \\ Hideki Tanaka \\ Okayama University, htanakaa@cc.okayama-u.ac.jp \\ Xiao Cheng Zeng \\ University of Nebraska-Lincoln, xzeng1@unl.edu
}

Follow this and additional works at: https://digitalcommons.unl.edu/chemzeng

Part of the Chemistry Commons

Slovak, Jan; Koga, Kenichiro; Tanaka, Hideki; and Zeng, Xiao Cheng, "Confined water in hydrophobic nanopores: Dynamics of freezing into bilayer ice" (1999). Xiao Cheng Zeng Publications. 57.

https://digitalcommons.unl.edu/chemzeng/57

This Article is brought to you for free and open access by the Published Research - Department of Chemistry at DigitalCommons@University of Nebraska - Lincoln. It has been accepted for inclusion in Xiao Cheng Zeng Publications by an authorized administrator of DigitalCommons@University of Nebraska - Lincoln. 


\title{
Confined water in hydrophobic nanopores: Dynamics of freezing into bilayer ice
}

\author{
Jan Slovák, ${ }^{*}$ Kenichiro Koga, ${ }^{\dagger}$ and Hideki Tanaka \\ Department of Chemistry, Faculty of Science, Okayama University, 3-1-1 Tsushimanaka, Okayama 700-8530 Japan \\ Xiao C. Zeng \\ Department of Chemistry, University of Nebraska-Lincoln, Lincoln, Nebraska 68588
}

(Received 3 June 1999)

\begin{abstract}
Molecular dynamics simulations for a thin film of water confined to a slit nanopore are performed in order to investigate the dynamic process of crystallization of the system. The system upon freezing creates a bilayer ice crystal composed of two layers of hexagonal rings. We perform one simulation at $T=257 \mathrm{~K}$ during which the system remains a supercooled liquid state, and another one at $T=253 \mathrm{~K}$ during which the system freezes. Many patterns of molecular arrangement are found upon freezing, and an account is given of the origin of multiple peaks in the distributions of binding energy and pair interaction energy. A definition of the solidlike cluster is introduced in order to analyze the time evolution of the clusters' population and their shapes. A large variety of shapes including highly nonspherical ones can be detected during simulations. A steady population of clusters is found at $T=257 \mathrm{~K}$, whereas at $T=253 \mathrm{~K}$ a post-critical nucleus of the solid phase emerges within a few nanoseconds and continues to grow until the system freezes completely. [S1063-651X(99)13411-1]

PACS number(s): 64.70.Ja, 61.20.Ja
\end{abstract}

\section{INTRODUCTION}

The interaction between water molecules, though mathematically simple in most model potentials, is responsible for its numerous anomalous properties and the large variety of structures that can be found under various thermodynamic conditions. One of the most ostentatious manifestations of the interaction is, of course, the hydrogen bond (HB) rendering to water molecules the ability of associating by themselves. To date, 12 kinds of crystalline ice structures have been experimentally established, and still newer ones are advocated to be uncovered. The last one, ice XII, was discovered quite recently in the unstable region of the ice $\mathrm{V}$ [1]. Experimental evidence was also found for two amorphous water structures, the so called LDA (low density amorphous) and HDA (high density amorphous) ices [2].

Apart from experimental observations, Monte Carlo and molecular dynamics (MD) computer simulations are very powerful tools to obtain deeper insight into the complex behavior of real substances. For systems with a complicated intermolecular interaction, the complexity of intermolecular potentials used in the simulations should always compromise with available computer performance. Therefore, to reproduce the essential thermodynamic behavior, the structure, and (eventually) some of the anomalies, and to give an account of their origin, one has to simplify considerably the real intermolecular potentials and bring them into more tractable forms. Effective pair potentials like simple point charge (SPC) [3] and a series of transferable intermolecular poten-

\footnotetext{
*Also at E. Hála Laboratory of Thermodynamics, Institute of Chemical Process Fundamentals, Academy of Science, 16502 Prague 6, Czech Republic.

${ }^{\dagger}$ Also at Department of Chemistry, Fukuoka University of Education, Munakata, Fukuoka 811-4192, Japan.

ॠAuthor to whom all correspondence should be addressed.
}

tials (TIP) [4,5] models are examples of the most reliable water effective potentials, and have been used successfully in water simulations under various conditions. One advantage of computer simulations is that they allow us to study even some metastable states (like LDA and HDA), most of which emerge under conditions hardly attainable or never tested in laboratory experiments, and thereby completely new structures and new features can be found.

The properties of water confined in nanopores can be dramatically different from their bulk counterparts and remain largely unexplored. Recently the transferable intermoleculars potential $4 \mathrm{P}$ (TIP4P) potential was used in an MD simulation of the liquid-solid phase transition of water confined in a narrow slit with hydrophobic walls [6]. The width of the slit was about $1 \mathrm{~nm}$, just enough to accommodate two layers of water molecules at a certain density. When the temperature of the system exposed to a fixed load (normal pressure in the direction perpendicular to the walls) is lowered, it undergoes a phase transition from a liquid to a solid phase. It was shown [6] in detail that the resulting phase is really a crystalline and not an amorphous one. The system forms a bilayer ice crystal, whose structure is unprecedented in the sense that it resembles none of the known structures of the ice polymorphs. Nevertheless the crystal still retains the basic feature of water ices-every molecule is attached by hydrogen bonding to four neighboring molecules. Each layer is composed of slightly distorted hexagonal rings (but still very flat unlike those in ice $\mathrm{I} h$ or Ic), both layers being completely in registry.

The original simulations were performed at loads of 50 $\mathrm{MPa}, 150 \mathrm{MPa}$, and $1 \mathrm{GPa}$ and the solid phase had the same crystalline structure in all three cases. It is more difficult and time consuming to simulate the phase transition under lower pressures since the transition temperature moves to the lower side with decreasing pressure. It is also shown that the bilayer ice, once created, remains stable after removing the walls, at least on the computer simulation time scale. The bilayer ice crystal also provides a somewhat new type of water surface. Normally water molecules on an ice surface 
are hydrogen bonded to at most three other water molecules. In addition, the outermost molecules are expected to be distorted heavily and thus have large vibrational amplitudes [7]. In contrast, every molecule in the bilayer ice has always four hydrogen bonded neighbors, which makes the surface structure exceptional. It is well known that water surfaces play a dominant role in many chemical reactions (e.g., as catalysts), and one can only guess what the consequences of the molecular arrangement in the bilayer ice surface would be on the nature of physical phenomena and chemical reactions in its vicinity. In the present work, we establish a relation between the arrangement of molecules and some thermodynamic properties in bilayer ice in order to extend our previous work [6] and to prepare for a study of nucleation, which is the main objective of future study.

\section{SIMULATION AND STRUCTURE ANALYSIS}

\section{A. Simulation}

We perform MD simulations at a fixed temperature $(T)$ and fixed load $1 \mathrm{GPa}\left(P_{z z}\right.$, normal pressure) on two plane parallel solid walls which confine a thin film of water. The equations of motion are integrated using a fifth order Gear predictor-corrector algorithm. The time step used is $0.5 \mathrm{fs}$, and the temperature and normal pressure are constrained using a Nosé-Andersen thermostat and barostat [8,9]. The time scales associated with the thermostat and barostat are $2-4 \mathrm{ps,}$ which are much smaller than the whole simulation time. The water molecules interact with each other via TIP4P potential and with the walls via the 9-3 Lennard-Jones potential. The long-range water-water potential is smoothly truncated at $8.665 \AA$. The simulation cell is a rectangular prism with lateral dimensions $l_{x}=65.01 \AA$ and $l_{y}=65.684 \AA$, thus keeping the area density $\rho_{A}=N / l_{x} l_{y}$ fixed. Our system consists of a fixed number of water molecules, $N=896$, which is considerably large compared to the previous simulation with 192 molecules [6]. Periodic boundary conditions are imposed in the $x$ and $y$ directions. The reason for increasing the size of the system is twofold: First, we want to show clearly that the phase transition is not an artifact of a small system size, and that the water film freezes in a larger system as well; second, we wish to gain some insight into the homogeneous nucleation process. It has been shown $[10,11]$ that the induction time and critical nucleus size depend strongly on the simulated system size. Swope and Andersen concluded that at least 15000 atoms were required to describe correctly the kinetics of crystal growth from a melt. They used a threedimensional system composed of atoms interacting via Lennard-Jones (LJ) interaction. Our system is quasi twodimensional, and from this point of view a size of about 1000 molecules is sufficient to remove the artifact arising from the small system size and the periodic boundary conditions, although the TIP4P interaction is not as simple as the $\mathrm{LJ}$ interaction and thus there is no a priori warranty to obtain a similar conclusion for our system.

\section{B. Definition of a solidlike cluster}

For the sake of a description of crystal growth and dissolution, we should identify solid phase embryos during the simulation. This is one of the central problems in computer

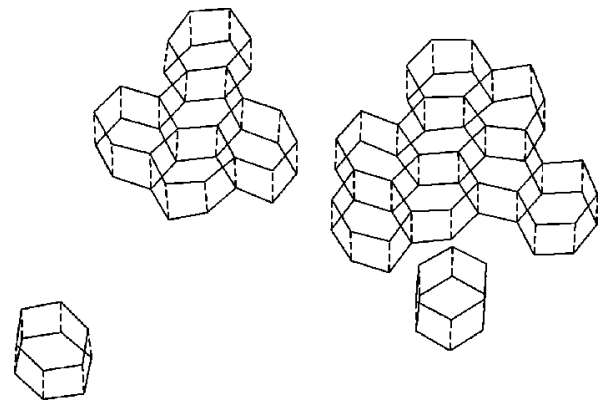

FIG. 1. An example of spatial arrangement of the molecules in the clusters due to our cluster definition. The solid lines depict the hydrogen bonds in each layer, the dashed ones are HB connecting molecules in different layers. The HB length is $2.73 \pm 0.02 \AA$.

simulation of a nucleation process and the kinetics of crystal growth. It presents a difficult task even for relatively simple systems like LJ liquids. Swope and Andersen, in their pioneering work, combined the steepest descent method with a Voronoi polyhedra analysis to identify solid regions in supercooled LJ liquid [11]. Both techniques are computer time demanding, so that, although appropriate for examining important aspects of the cooling process, it would be difficult to gather sufficient statistical data for a detailed investigation of nucleation.

An alternative method was suggested for a standard solid region recognition criterion [12], where use is made of internal angles of triplets of atoms. Criteria of this type can be used appropriately during simulations in order to study the cluster dynamics of crystal growth. It is, however, good to keep in mind that various definitions of clusters do not necessarily give exactly the same cluster sizes and shapes. It generally holds that the simpler the criterion used in the definition of clusters, the more defects are observed.

We cannot simply define solidlike and liquidlike molecules in terms of single molecule characteristics. The binding energy of a molecule, the number of its hydrogen bonds, the numbers and positions of its nearest neighbors, are not enough to characterize the phase (state) of the water molecules. Triplets of molecules do not seem to be a promising alternative either. Nevertheless, it is clear that the assembly of "coupled" hexagonal rings is exactly what makes the thin film a bilayer ice. We will call a pair of hexagons, i.e., a hexagonal ring in one layer with its complement in another layer, a hexagonal cell. One hexagonal cell seems to be the smallest unit having solidlike features, so we will regard it as the smallest embryo of the solid phase. A solid crystal now grows by attaching more hexagonal cells, not necessarily one after another. Figure 1 shows an example of a configuration during freezing, which contains four clusters of sizes $8,5,1$, and 1.

All the molecules in a solid cluster fulfil two simple presumptions: (1) each molecule has four hydrogen bonded neighbors (hereafter called species-4 molecules), and (2) each pair of hydrogen bonded molecules belong to at least one hexagonal ring of hydrogen bonded molecules. A cluster is defined as a maximally connected (via HB) set of molecules satisfying these two rules. The boundary of a cluster is a very complicated region. We designate that a cluster molecule is a boundary molecule unless all four nearest neighbors belong to the cluster. Let us call these nearest neighbors 

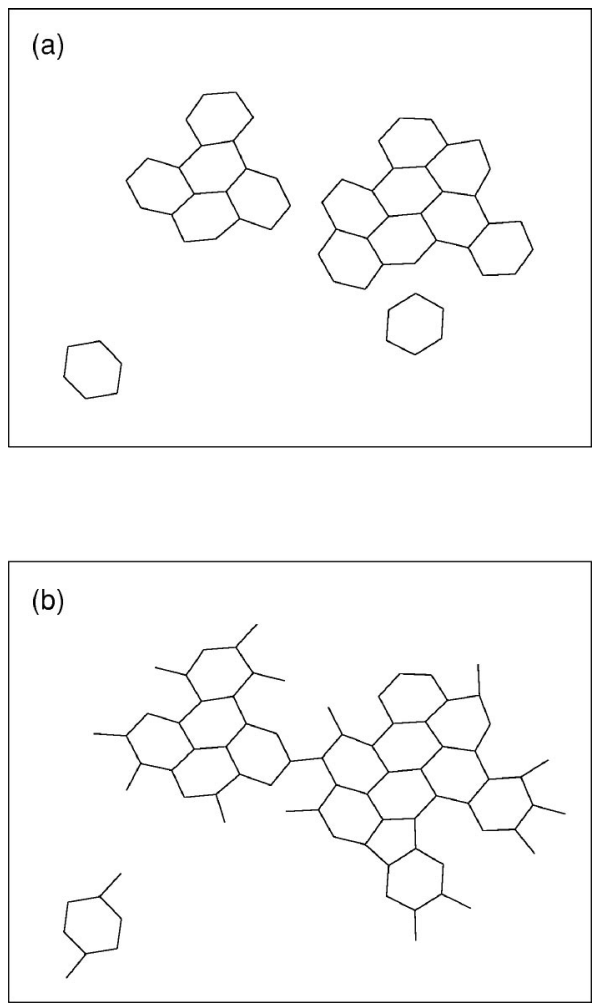

FIG. 2. An example of solid clusters ( $x y$ projection) considering two definitions of a cluster. See the text for explanation.

of a boundary molecule, which do not belong to the cluster, interfacial molecules. Among the interfacial molecules, some are species 4 like the cluster molecules, and others are not. We could slightly modify our cluster definition by including species-4 interfacial molecules into the cluster. For some purposes, the modified definition of the cluster could turn out to be more appropriate than the original one. Figure 2(b) shows the result after applying the modified definition, whereas Fig. 2(a) depicts the situation according to the original rules. It is the same configuration as that in Fig. 1. (From now on we employ the $x y$-plane projection of the configurations as their graphical representation.) The cluster shapes can change dramatically upon applying the modified definition. While there are four clusters in Fig. 2(a), there are only two clusters in Fig. 2(b), the original 8- and 5-clusters being connected by double HB bridge and the 1-cluster connected to 8-cluster via an intermediate pentagonal cell. There is no reason a priori to adhere to one definition, and it could be slightly modified in many other ways in so far as it characterizes ice nuclei in our system.

\section{RESULTS AND DISCUSSION}

We perform two sets of MD simulations, and examine nuclei growth in subsequent analyses. The first set is a strongly supercooled liquid at a temperature of $257 \mathrm{~K}$. The simulation time is $10 \mathrm{~ns}$, and during this time the system remains in metastable equilibrium without freezing. Then the temperature is decreased to $253 \mathrm{~K}$ and we perform the second simulation for $12 \mathrm{~ns}$, toward the end of which the system freezes into a bilayer ice crystal.

For the sake of analysis, we store every thousandth configuration, the time step being $5 \times 10^{-16} \mathrm{~s}$. These configura-

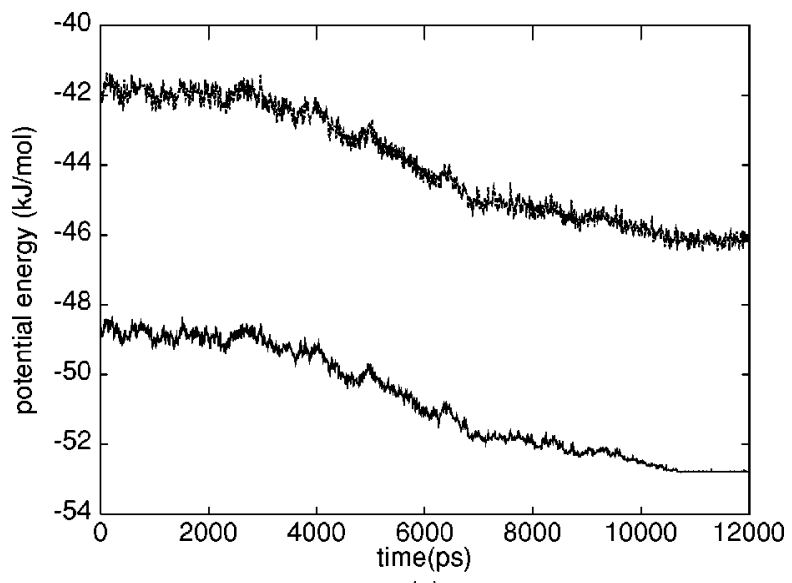

(a)

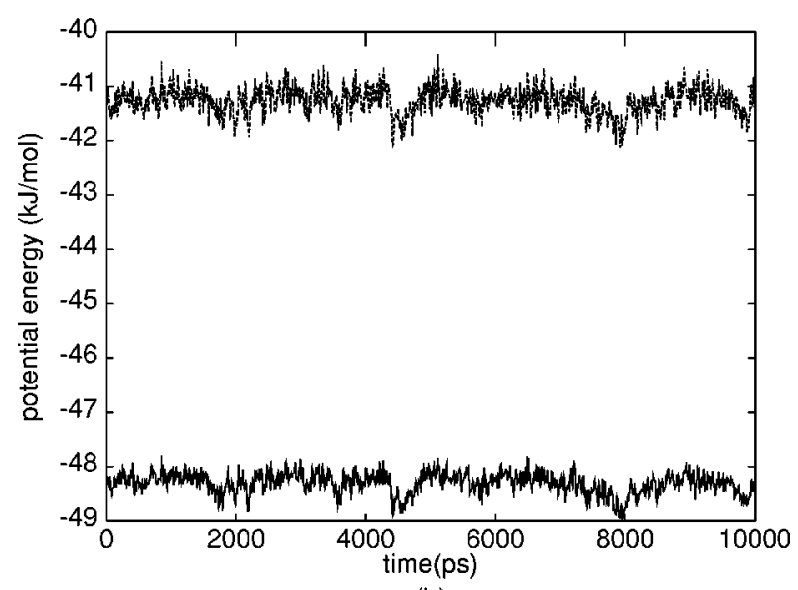

(b)

FIG. 3. The potential energy (without the interaction with the walls) as a function of time for $T=253 \mathrm{~K}$ (a) and $T=257 \mathrm{~K}$ (b). The solid line is for quenched structures, and the dashed line for instantaneous structures.

tions, obtained in the run, are called instantaneous $(I)$ structures. The potential energy for $I$ structures is composed of two contributions: the potential energy of minimum energy structures and a contribution due to the thermal energy. The minimum energy structures correspond to stable points in configuration space, and therefore are free of thermal excitations $[13,14]$. Those are referred to as quenched $(Q)$ structures [15]. To obtain $Q$ structures from $I$ structures, we use the steepest descent method. From $I$ structures, various thermodynamic and kinetic properties are usually calculated. On the other hand, $Q$ structures can provide useful information about the structure in configuration space and the geometry of molecular arrangement, especially for the solid state.

The potential energies as functions of simulation time are plotted in Fig. 3 for $T=253 \mathrm{~K}$ (a) and $T=257 \mathrm{~K}$ (b). The potential energy includes only water-water interactions; the wall-water interactions are removed. It can be seen that the energy fluctuates around a mean value for $T=257 \mathrm{~K}$ and falls substantially during freezing at $253 \mathrm{~K}$. The potential energy $U_{\text {int }}=-41.27 \pm 0.25 \mathrm{~kJ} / \mathrm{mol}$ for $T=257 \mathrm{~K}$ in the $I$ structure and $U_{\mathrm{int}}=-48.31 \pm 0.20 \mathrm{~kJ} / \mathrm{mol}$ in the $Q$ structure, and $U_{\mathrm{int}}=-46.13 \pm 0.13 \mathrm{~kJ} / \mathrm{mol}$ for $T=253 \mathrm{~K}$ in the $I$ structure and $U_{\text {int }}=-52.78 \pm 0.01 \mathrm{~kJ} / \mathrm{mol}$ in the $Q$ structure. The energy corresponding to $Q$ structures is always lower by 6-7 $\mathrm{kJ} / \mathrm{mol}$ than that of $I$ structures, but it follows a similar time 


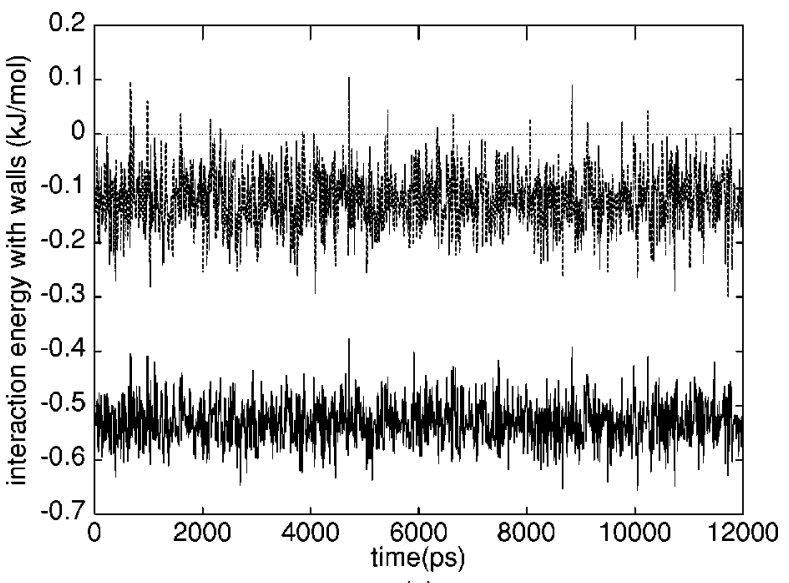

(a)

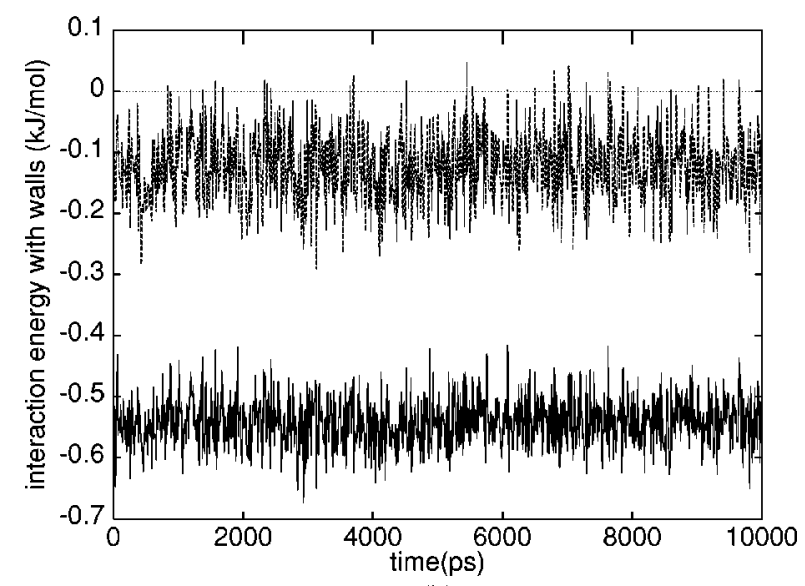

(b)

FIG. 4. The interaction energy of the system with walls as a function of time for $T=253 \mathrm{~K}$ (a) and $T=257 \mathrm{~K}$ (b). The solid line is for quenched structures, and the dashed line for instantaneous structures.

evolution, i.e., the local minima and maxima on the $I$ and $Q$ curves match each other. Those local extremes mostly correspond to changes in spatial distribution of clusters as well as changes in their sizes. Figure 4 shows the time evolution of the water-wall interaction (per water molecule). It is evident that the potential energy arising from the water wall is not significant at any stage of crystallization. No qualitative or quantitative changes in the water-wall interaction energy accompanying the phase change are observed. Actually this is quite an interesting observation, because there is a definite change in wall to wall distance in the course of freezing (see Fig. 5). However it seems that the change in the wall-wall distance and the change in the structure of confined water are mutually correlated, and the water-wall potential energy remains constant in its mean value.

Figure 6 shows the distribution of the binding energy of molecules (i.e., the total interaction energy of a molecule with its surroundings). The binding energy distribution for quenched solid structures shows double peak [solid curve in Fig. 6(a)]; otherwise all the distribution curves are unimodal Gaussian-like. Figure 7 displays the pair interaction energy distribution for water molecules. There is a large gap in the distribution curve for $Q$ structures at $T=253 \mathrm{~K}$, approximately between -15 and $-10 \mathrm{~kJ} / \mathrm{mol}$. There are four clearly distinguishable peaks in the low energy region below -15

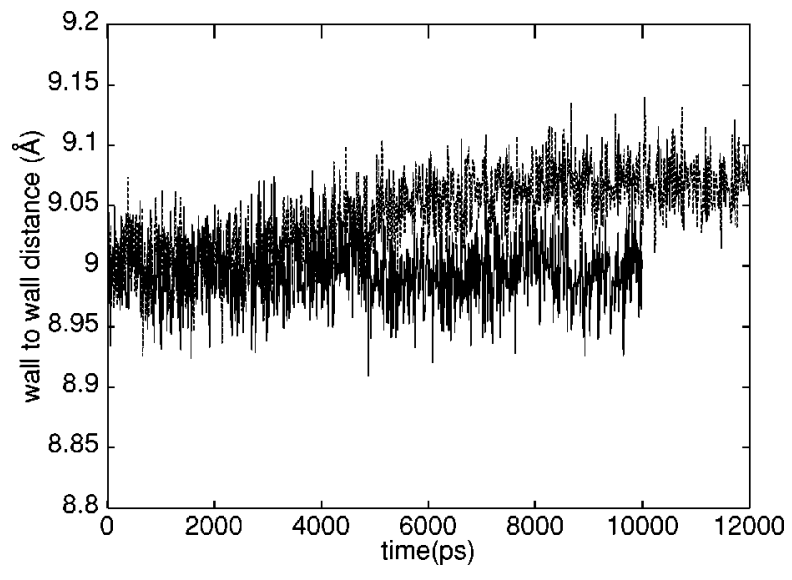

FIG. 5. Wall to wall distance as a function of time for $T$ $=257 \mathrm{~K}$ (solid line) and $T=253 \mathrm{~K}$ (dashed line).

$\mathrm{kJ} / \mathrm{mol}$. There are only three distinguishable peaks in hexagonal ice and only two peaks in cubic ice in TIP4P water [16]. Those correspond to pairs of hydrogen bonded molecules. It is thus natural to adopt $u_{\mathrm{HB}} \leqslant-15 \mathrm{~kJ} / \mathrm{mol}$ as an energetic criterion for hydrogen bond. We call a pair of molecules whose interaction energy satisfies this condition a hydrogen bonded pair. This is valid only when analyzing $Q$

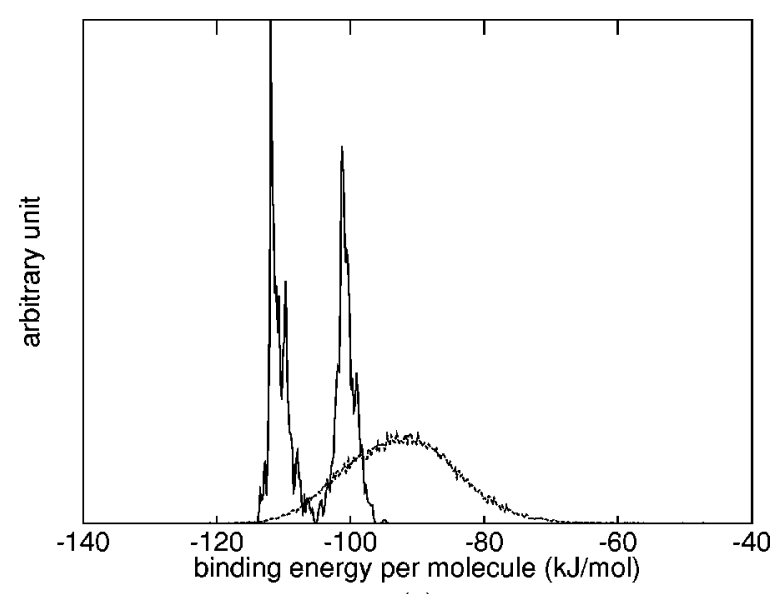

(a)

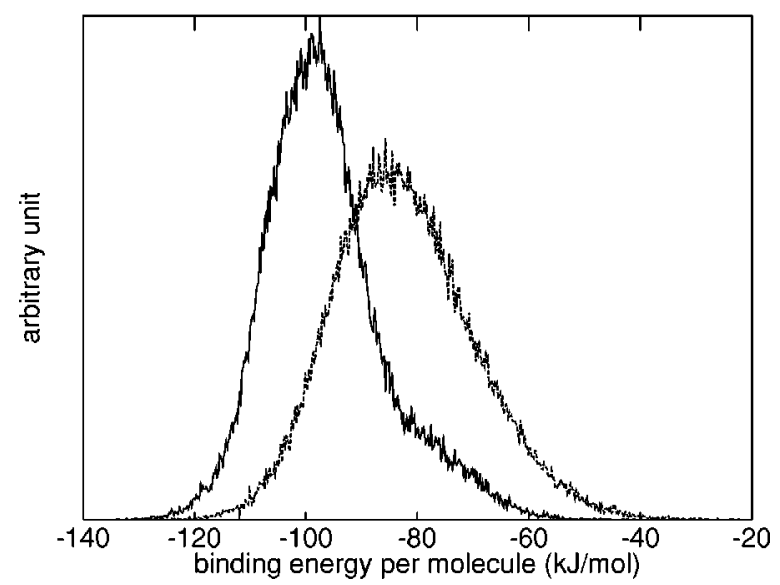

(b)

FIG. 6. The binding energy distribution per molecule for $T$ $=253 \mathrm{~K}$ (a) and $T=257 \mathrm{~K}(\mathrm{~b})$. The solid line is for quenched structures, and the dashed line for instantaneous structures. 


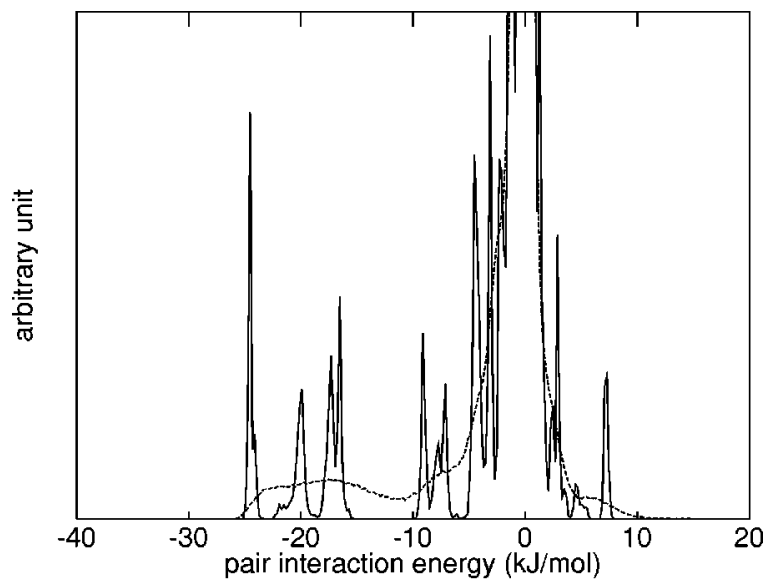

(a)

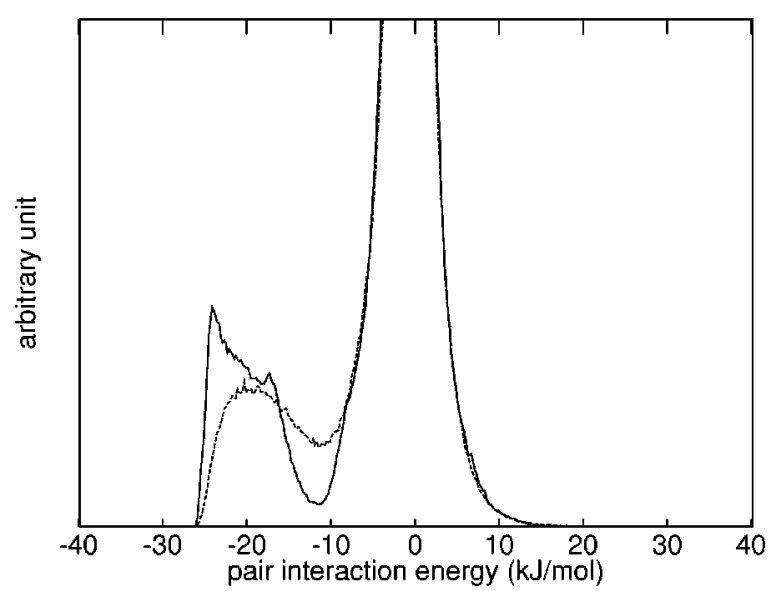

(b)

FIG. 7. The pair interaction energy distribution for $T=253 \mathrm{~K}$ (a) and $T=257 \mathrm{~K}(\mathrm{~b})$. The solid line is for quenched structures, and the dashed line for instantaneous structures.

structures, but it would not give correct results for $I$ structures. There is another small gap in between -6.5 and -5.5 $\mathrm{kJ} / \mathrm{mol}$. A pair interaction energy lower than $-6.5 \mathrm{~kJ} / \mathrm{mol}$ corresponds to an interaction with the second neighbors of a chosen molecule lying in the opposite layer. Every molecule has four nearest neighbors and nine second nearest neighbors, three of them lying in the opposite layer. The interaction energy between a central molecule and one or two of the three second nearest neighbors in the opposite layer is below the second gap. Statistically the number of molecules having one such neighbor is approximately the same as the number of molecules having two. The remaining molecules among three in the opposite layers have an interaction energy with a chosen molecule higher than the gap position. In this way, molecules can be divided into two classes with respect to the number of second nearest molecules having a lower interaction energy than $-6.5 \mathrm{~kJ} / \mathrm{mol}$. This also gives a rough explanation of the double peak in the binding energy distribution. Molecules belonging to different classes have lower (left peak) or higher (right peak) binding energies. Figure 8 shows a typical example of the distribution of water molecules in one layer into two classes, each with a different binding energy. The complementary molecules belong to different classes (with very rare exceptions which can be viewed as defects). Another fact worth mentioning is that after the sys-

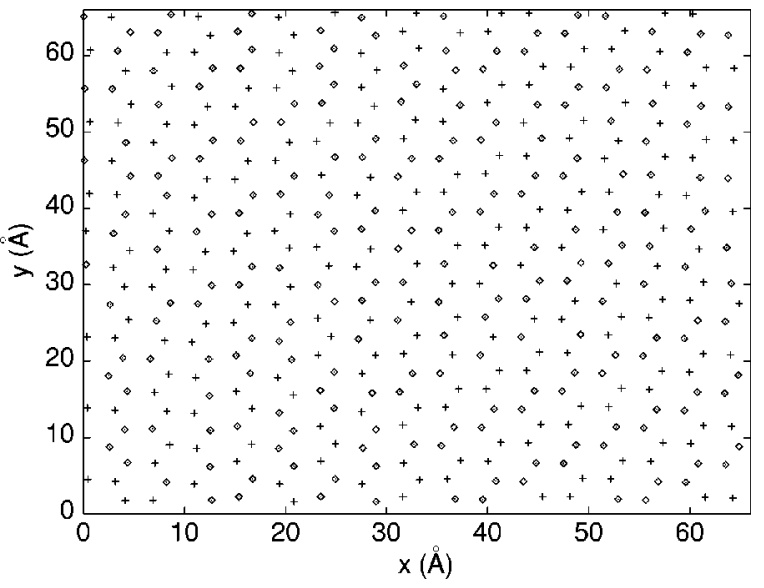

FIG. 8. The arrangement of the molecules with the higher and lower binding energies (corresponding to the second and first peak on the binding energy distribution curve) in one of the layers after freezing at $t=12 \mathrm{~ns}$.

tem crystallizes, i.e., during the last $2 \mathrm{~ns}$, the distribution of molecules to the two classes never changes. It is worthwhile restating the extra rules discovered for bilayer ice [6]. Hexagonal rings are not regular but have three different $\mathrm{O}-\mathrm{O}-\mathrm{O}$ angles: $\alpha=108^{\circ}, \beta=118.5^{\circ}$, and $\gamma=133.5^{\circ}$ (all within error bar $\pm 2^{\circ}$ ). The bilayer rules are as follows: (1) $\mathrm{H}-\mathrm{O}-\mathrm{H}$ bond angle can be superimposed only over the $\alpha$ angle; and (2) when one $\mathrm{OH}$ arm of a water molecule is normal to the hexagonal lattice plane, the other $\mathrm{OH}$ arm can point only along a direction adjacent to the $\alpha$ angle edges. In accordance with these rules, once the crystal is created, the rearrangement of hydrogen atoms leading to the change of class of a molecule would require a simultaneous orientational rearrangement of at least four molecules [6]. This kind of rearrangement is not plausible under the simulated conditions. A detailed explanation of the origin of all multiple peaks in the binding energy and pair interaction energy distributions is difficult because of the high local asymmetry, which results in so many different $\mathrm{O}-\mathrm{O}-\mathrm{O}$ angles and a mutual orientation of neighboring molecules. This is in contrast to a similar and feasible analysis for hexagonal or cubic ices, where at most four dihedral angles can be observed, in terms of which the account for the origin of peaks can be given [16]

We also perform a normal mode analysis for $I$ structures, and calculate the number of imaginary frequency modes for $I$ structures. Some of these modes are unstable modes, and play an important role in diffusive motions, although the relation has not yet been established qualitatively $[17,18]$. The number of imaginary modes is approximately 491 out of 5373 total modes $(8.9 \%)$ during simulation at $257 \mathrm{~K}$. At 253 $\mathrm{K}$, the number of imaginary modes decreases from about $430-440(8.1 \%)$, at the beginning of the simulation, to about $250(4.7 \%)$ at the end.

During the simulations we follow the size and distribution of solidlike clusters. To find the clusters, we use the $Q$ structures and cluster definition from Sec. II. Following the cluster distribution as a function of time, we sometimes observe very abrupt changes even during a short time (several tens of simulation steps), and sometimes a cluster seems to be stable for quite a long time. Figure 9 shows an example of the time 

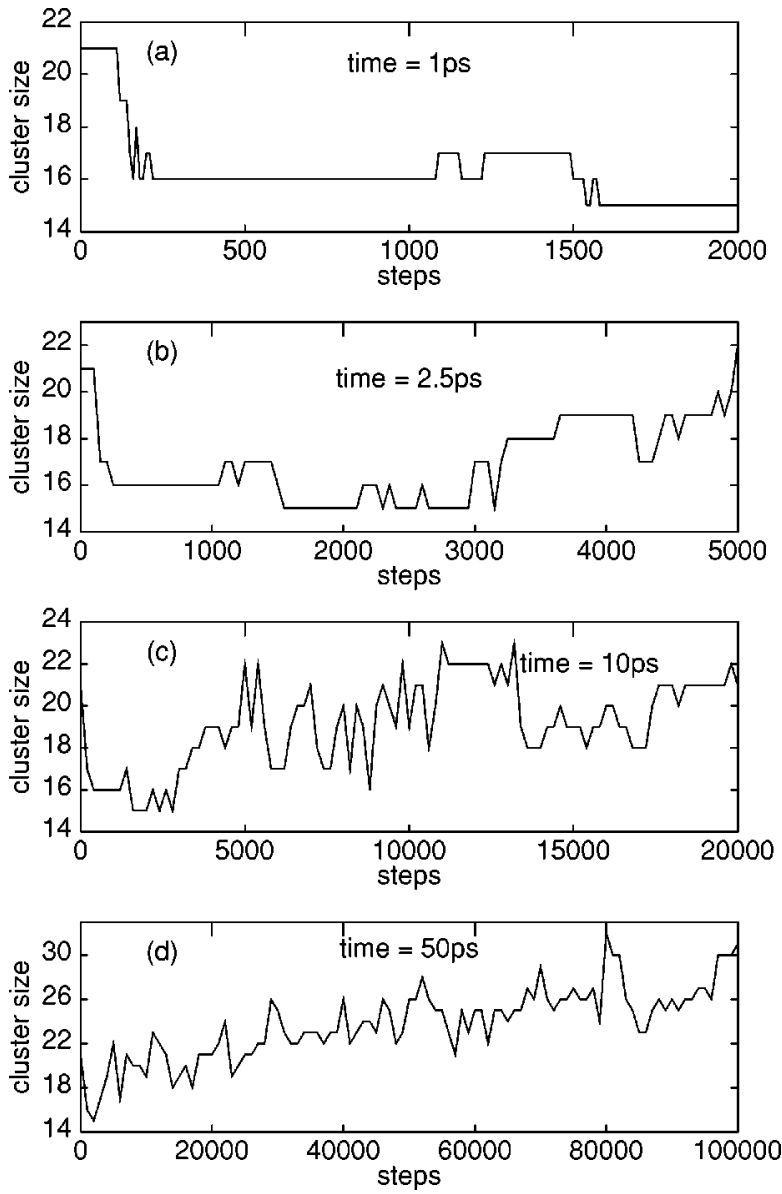

FIG. 9. The time dependence of a cluster size for various time scales at the temperature $T=253 \mathrm{~K}$.

evolution of a maximal size cluster at $T=253 \mathrm{~K}$ on various time scales. We start with a configuration containing a maximal cluster size equal to 21 . It can be seen that the cluster size remains unchanged sometimes even for $0.2-0.5 \mathrm{ps}$, but we never observe a longer stabilization. Similar results to those for stability are obtained at $T=257 \mathrm{~K}$. The cluster in Fig. 9 has a tendency to grow, which becomes clearer on large time scale plots. Over 50 ps the cluster size increases from 21 at the beginning to 31 at the end. Other clusters are also present during simulation. Usually there are up to 5-6 smaller clusters of sizes ranging from 1 to 4 . Larger ones are never observed. The lifetime of the small clusters is approximately of the order of 1-10 ps; they appear and disappear again.

Figure 10 shows the time evolution of the maximal cluster size during simulations. In the liquid, we occasionally detect some large clusters (17-19) but they always melt and never serve as a nucleus for the crystal phase. The lifetime of all the clusters is definitely shorter than the total simulation time (10 ns). In a colder system, we observe the onset of crystallization at about $t=5 \mathrm{~ns}$. It is evident that the cluster rapidly grows, and crystallization takes place. The nucleus itself is already apparently present at $t \approx 2 \mathrm{~ns}$. The dashed curve shows the size of the second largest cluster. At $t \approx 4.2 \mathrm{~ns}$ the two largest clusters of about the same size (20) join and create a large cluster which continues to grow. The dashed curve does not correspond to only one specific cluster and its dynamics, but simply shows the size of the second largest

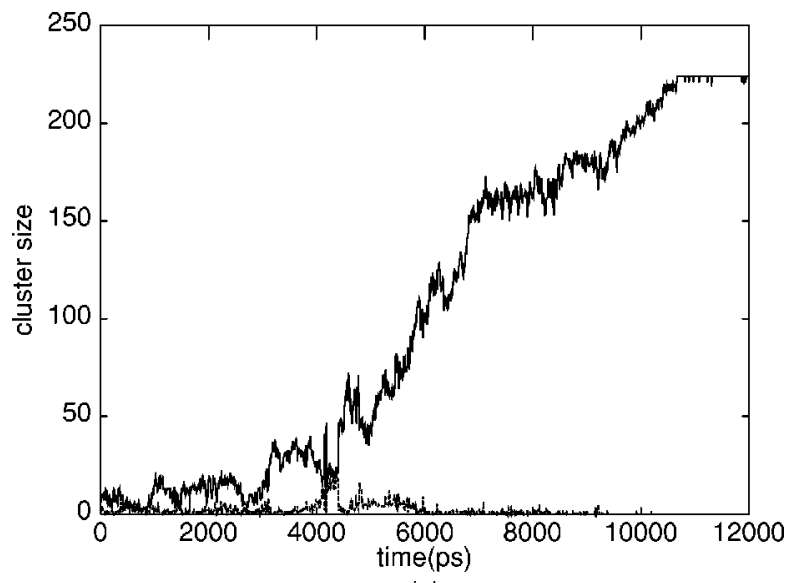

(a)

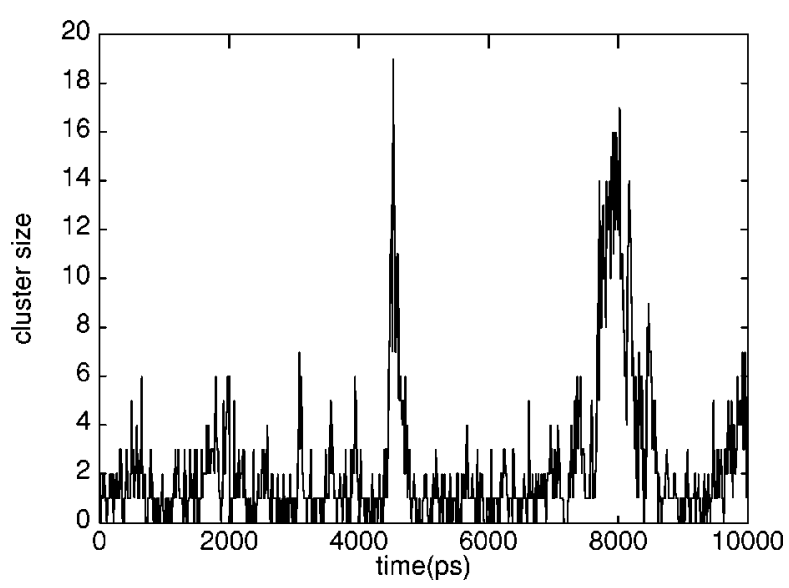

(b)

FIG. 10. The maximum cluster size as a function of time for $T=253 \mathrm{~K}$ (a) and $T=257 \mathrm{~K}$ (b). The dashed line in picture (a) is for the time dependence of the second largest cluster size.

cluster at a given time. One such cluster can be replaced by another one.

The shape of the clusters deserves special comment. We follow the exact shape of the clusters during the liquid simulation. It seems that there is no preference of spherical shape over any other shape (actually by "spherical" we mean circular or spherical in two and three dimensions; hereafter we will call shapes spherical). Nearly spherical clusters can be observed as well as very nonspherical ones. Generally, if we identify the hypothetical center of a cluster (one of its hexagons) and its maximal spherical subcluster, then the whole cluster often contains molecules even in the second and third shells (made from hexagons) which surround the spherical subcluster. Sometimes something like a double cluster is detected, which means a cluster formed by two subclusters joined by a one cell bridge. We also observe some signs of dynamical effects such as translational and rotational movement of whole clusters (though it looks rather like vibrations than a long distance shift). As for smaller clusters, the same diversity in shapes can be observed for both temperatures. At $T=253 \mathrm{~K}$, other interesting shapes can be observed for larger clusters. The shape of clusters can be also roughly characterized by a single parameter of asphericity which can be defined as

$$
\eta=\frac{l^{2}}{4 \pi S}
$$


TABLE I. Asphericity $\eta$ for the clusters detected during the simulation at $T=253$ and $257 \mathrm{~K} . \eta_{\min }$ is the lowest asphericity detected for a given cluster size. $\eta_{\max }$ the highest asphericity and $\eta_{\mathrm{av}}$ the average.

\begin{tabular}{lcccccc}
\hline \hline & \multicolumn{3}{c}{$T=253 \mathrm{~K}$} & \multicolumn{3}{c}{$T=257 \mathrm{~K}$} \\
\cline { 2 - 7 } Size & $\eta_{\min }$ & $\eta_{\max }$ & $\eta_{\text {av }}$ & $\eta_{\min }$ & $\eta_{\max }$ & $\eta_{\text {av }}$ \\
\hline 1 & 1.12 & 1.12 & 1.12 & 1.12 & 1.12 & 1.12 \\
2 & 1.55 & 1.55 & 1.55 & 1.55 & 1.55 & 1.55 \\
3 & 1.49 & 2.03 & 1.83 & 1.49 & 2.03 & 1.78 \\
4 & 1.52 & 2.52 & 1.94 & 1.52 & 2.52 & 2.04 \\
5 & 1.59 & 3.01 & 2.11 & 1.59 & 3.01 & 2.13 \\
6 & 1.68 & 3.50 & 2.34 & 1.68 & 3.50 & 2.22 \\
7 & 1.77 & 3.48 & 2.64 & 1.77 & 3.00 & 2.17 \\
8 & 1.55 & 3.50 & 2.60 & 1.55 & 3.05 & 2.09 \\
9 & 1.99 & 4.48 & 2.93 & 1.67 & 3.11 & 2.38 \\
10 & 1.79 & 4.49 & 3.02 & 1.79 & 3.60 & 2.51 \\
11 & 1.63 & 3.27 & 2.56 & 1.91 & 3.66 & 2.77 \\
12 & 1.75 & 4.57 & 2.84 & 1.75 & 3.74 & 2.36 \\
\hline
\end{tabular}

where $S$ is the surface of a cluster's $x y$ projection, and $l$ is the length of its boundary. By definition $\eta$ is 1 for a circle. Table I lists the asphericities $\eta$ of the clusters up to size 12 detected during the simulations at the both temperatures. The asphericities of the clusters seem to be more or less the same for both temperatures. The asphericity of the most spherical shapes for a given size of cluster fluctuates in the range 1.44-1.68 for sizes up to 70 . The linear chain of hexagonal units is the cluster with the highest asphericity.

Figure 11 shows examples of clusters, observed at $T$ $=253 \mathrm{~K}$. The system at $t=3.2 \mathrm{~ns}$ [Fig. 11(a)] contains an embryo of a rather elliptical (or renal) shape with $\eta=3.87$. Large clusters gradually grow more like a coral than a pearl. The cluster in Fig. 11(b) ( $t=3.93 \mathrm{~ns})$ looks rather spherical, but contains a hole which makes its asphericity quite large $(\eta=3.27)$. In the early stage of simulation when only small clusters are present, the chance to observe more spherical clusters is higher and the clusters possess a rich variety of shapes [Figs. 11(g) and 11(h)]. Figure 11(c) shows two relatively large clusters joined by a one cell bridge $(t=4.17 \mathrm{~ns}$, $\eta=7.95$ ). Figure $11(\mathrm{~d})$ depicts the situation shortly before $(t=4.05 \mathrm{~ns})$. Clusters of size 60-80 contain various defects (small holes embedded in nonhexagonal rings). Such a defect can be seen in Fig. 11(b)'s configuration (with five and seven rings in the hole). Embryos of a size of about 80 still deserve to be called clusters, i.e., the system is not yet percolated. Interesting examples of embryo shapes are the configurations in Figs. 11(d) and 11(e) $(t=4.13 \mathrm{~ns})$, which have a fractallike structure. The configuration in Fig. 11(f) $(t=4.56 \mathrm{~ns}$, $\eta=9.27$ ) contains one large highly nonspherical cluster. To conclude, the nucleation is not spherical wrapping of one layer (spherical shell) onto another (not even on average). $A$ priori, there is no reason to expect spherical shapes. It is well known from measurements of $\mathrm{I} h$ crystal shapes in cirrus clouds that these crystals do have wide variety of shapes, including approximately equidimensional particles, columns, plates, bullet rosettes and irregular aggregates [19]. This does not necessarily mean that these shapes can be observed in the size of critical or slightly postcritical nuclei, but this is very
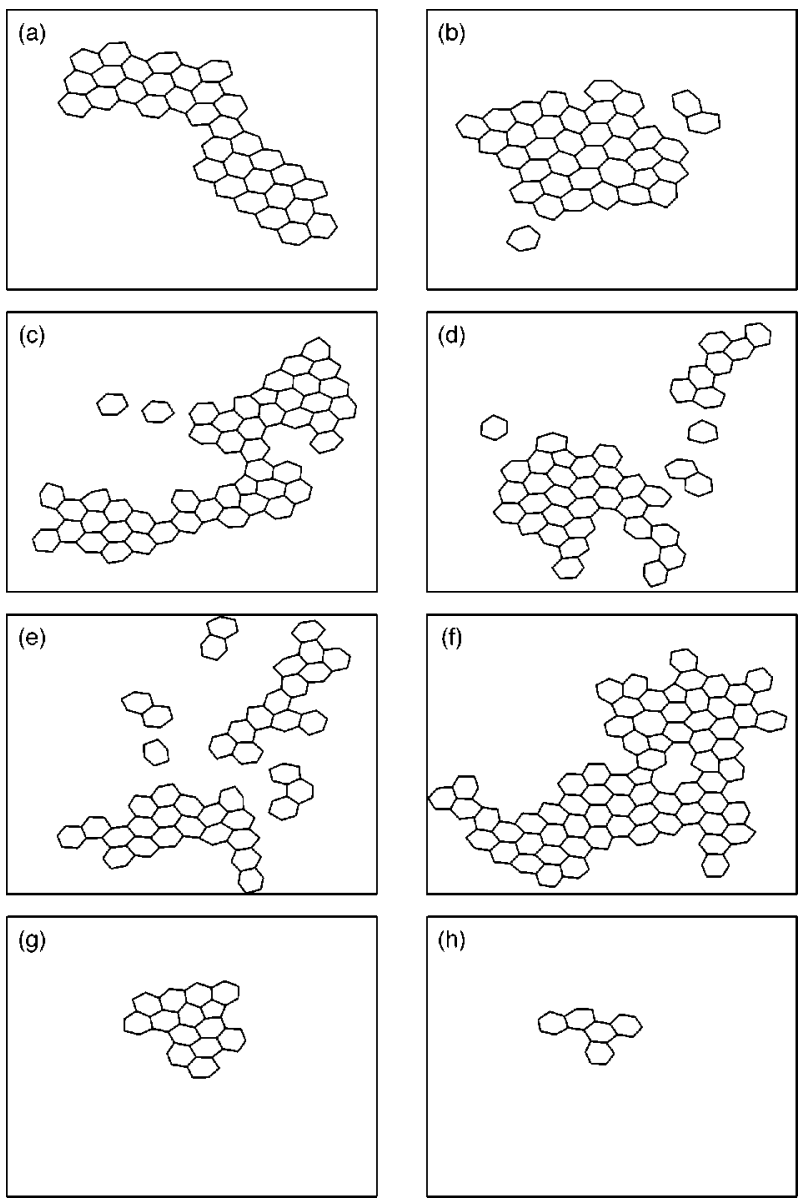

FIG. 11. Examples of solid clusters in the system during freezing. Various stages of crystal growth at $253 \mathrm{~K}$. Each rectangular box corresponds to the size of the simulation cell.

probable, especially because the size of the crystallites in the atmosphere is rather microscopic. One could expect a similar behavior for other ice polymorphs as well.

\section{CONCLUDING REMARKS}

MD simulations for a thin film of water confined to a slit nanopore are performed in order to investigate the dynamic process of crystallization of the system. Upon freezing the system creates a bilayer ice crystal composed of two layers of hexagonal rings, each molecule being connected by hydrogen bonds to four neighboring molecules. We detect many patterns of arrangement of the neighbors of a given molecule. This results, for example, in the division of molecules into two classes according to their binding energy corresponding to $Q$ structures.

We perform a detailed analysis of the dynamics of solid clusters, which includes a definition of a solidlike cluster; we then follow the process of growing and dissolving the clusters during freezing. A certain population of smaller clusters is found even for a liquid system at $T=257 \mathrm{~K}$, which indicates that the system is highly supercooled. During the phase transition at $T=253 \mathrm{~K}$ we follow the population and shapes of clusters, and it seems that there is no preference for spherical shapes; clusters with quite complicated topologies are observed as well. 
Having performed the above study we are now ready to start a study of nucleation which would include calculation of quantities like the nucleation rate and critical nucleus size. One problem could be the use of $Q$ structures in the cluster definition. Everything is all right unless we need to carry out cluster analysis too frequently. Then the cluster analysis (which requires quenching) becomes very time consuming; some other way of extracting a fundamental structure would be preferable. One of these ways is coarse graining. In this method the coordinates of molecules are averaged over some time $\Delta \tau$,

$$
\overline{\mathbf{r}}_{i}(t)=\int_{-\Delta \tau / 2}^{\Delta \tau / 2} \mathbf{r}_{i}\left(t+t^{\prime}\right) d t^{\prime}
$$

where $\mathbf{r}_{i}$ represents both translational and orientational coordinates. Thus obtained structures are often called $V$ structures $[20,21] . \Delta \tau \approx 500$ steps seems to be optimal choice. We tested this method on the system corresponding to Fig. 9(a) in a time interval of 300-1000 steps and the cluster sizes calculated from $V$ structures are about the same as those from $Q$ structures. For the largest cluster the difference in size obtained from both methods was two cells at most. Generally the coarse graining method can be less exact when the size of a cluster changes more frequently in a short time and more defects are expected. However, on the average the dynamics of cluster growth will be the same, no matter which fundamental structures are used. Coarse graining is much less time consuming and feasible, even when performed frequently. The two ways can eventually be combined.

\section{ACKNOWLEDGMENTS}

J.S. and H.T. are grateful to the Japanese Ministry of Education and Culture for support during visit to Japan, and a grant-in-aid for scientific research. The present work was also supported by NSF and JSPS.
[1] C. Lobban, J. L. Finney, and W. F. Kuhs, Nature (London) 391, 268 (1998).

[2] O. Mishima and E. Stanley, Nature (London) 396, 329 (1998).

[3] H. J. C. Berendsen, J. R. Grigera, and T. P. Straatsma, J. Phys. Chem. 91, 6269 (1987).

[4] W. L. Jorgensen, J. Chem. Phys. 77, 4156 (1982).

[5] W. L. Jorgensen, J. Chandrasekhar, J. D. Madura, R. W. Impey, and M. L. Klein, J. Chem. Phys. 79, 926 (1983).

[6] K. Koga, X. C. Zeng, and H. Tanaka, Phys. Rev. Lett. 79, 5262 (1997).

[7] N. Materer, U. Starke, A. Barbieri, M. A. Van Hove, G. A. Somorjai, G.-J. Kroes, and C. Minot, J. Phys. Chem. 99, 6267 (1995).

[8] S. Nosé, Mol. Phys. 52, 255 (1984).

[9] H. C. Andersen, J. Chem. Phys. 72, 2384 (1980).

[10] J. D. Honeycutt and H. C. Andersen, J. Phys. Chem. 90, 1585 (1986).
[11] W. C. Swope and H. C. Andersen, Phys. Rev. B 41, 7042 (1990).

[12] A. D. J. Haymet, Chem. Phys. Lett. 107, 77 (1984).

[13] F. H. Stillinger and T. A. Weber, J. Phys. Chem. 87, 2833 (1983).

[14] F. H. Stillinger and T. A. Weber, Science 225, 983 (1984).

[15] I. Ohmine, H. Tanaka, and P. G. Wolynes, J. Chem. Phys. 89, 5852 (1988).

[16] H. Tanaka and I. Okabe, Chem. Phys. Lett. 259, 593 (1996).

[17] J. D. Gezelter, E. Rabani, and B. J. Berne, J. Chem. Phys. 107, 4618 (1997).

[18] T. Keyes, J. Chem. Phys. 101, 5081 (1994).

[19] J. Jensen, O. B. Toon, D. L. Westphal, S. Kinne, and A. J. Heymsfield, J. Geophys. Res. 99, 10421 (1994).

[20] D. Eisenberg and W. Kauzmann, The Structure and Properties of Water (Oxford University, London, 1969).

[21] F. Hirata and P. J. Rossky, J. Chem. Phys. 74, 6867 (1981). 